\title{
A unipolarly located, cell-surface-associated agglutinin, RapA, belongs to a family of Rhizobium-adhering proteins (Rap) in Rhizobium leguminosarum bv. trifolii
}

\author{
Nora Ausmees, Karin Jacobsson and Martin Lindberg
}

Swedish University of Agricultural Sciences, SLU, Department of Microbiology, Box 7025 S-75007 Uppsala, Sweden
Author for correspondence: Nora Ausmees. Tel: +46186732 06. Fax: +4618673392. e-mail: nora.ausmees@mikrob.slu.se

The phage-display cloning technique was used to find rhizobial proteins that bind to receptors located on the bacterial cell surface. The aim was to clone the gene(s) encoding rhicadhesin, a universal rhizobial adhesion protein, and/or other cell-surface-binding proteins. Four such Rhizobium-adhering proteins (Rap) were revealed in Rhizobium leguminosarum bv. trifolii strain R200. The binding is mediated by homologous Ra domains in these proteins. One member of the Rap protein family, named RapA1, is a secreted calcium-binding protein, which are also properties expected for rhicadhesin. However, the size of the protein (24 kDa instead of $14 \mathrm{kDa}$ ) and its distribution among different rhizobia (present in only Rhizobium leguminosarum biovars and $R$. etli instead of all members of Rhizobiaceae) argue against RapA1 being rhicadhesin. Protein RapA1 consists of two homologous Ra domains and agglutinates R200 cells by binding to specific receptors located at one cell pole during exponential growth. Expression of these cell-surface receptors was detected only in rhizobia that produce the RapA proteins. The authors propose that the homologous Ra domains, found to be present also in other proteins with different structure, represent lectin domains, which confer upon these proteins the ability to recognize their cognate carbohydrate structures.

Keywords: rhizobia, lectin, calcium-binding protein, phage display

\section{INTRODUCTION}

Rhizobia are soil bacteria capable of fixing nitrogen in symbiosis with leguminous plants. During early stages of infection, these bacteria adhere to the root hairs of host plants. The adhesion appears to be a complex multistep process consisting of several interactions, both specific and non-specific. Several authors have described different host-specific attachment patterns in different rhizobia. The lectin-recognition hypothesis raised first by Dazzo \& Hubbell (1975) proposes that the specific attachment of homologous rhizobia to the host plant root hairs is dependent on the host plant lectin. The early adsorption of Rhizobium leguminosarum bv.

Abbreviations: CMC, carboxymethylcellulose; EPS, exopolysaccharide; PAA, particle agglutination assay; RA or Ra, Rhizobium-adhering (phage or domain).

The GenBank accession numbers for the sequences reported in this paper are AF265222, AF265223, AF315809 and AF315810. phaseoli to bean roots has also been shown to be hostspecific and dependent on the presence of $\mathrm{Ca}^{2+}$ and $\mathrm{Mg}^{2+}$ ions (Lodeiro et al., 1995). In Bradyrhizobium japonicum, a bacterial lactose-sensitive lectin located at one pole of the bacteria has been reported to determine the specific binding of the bacteria to soybean roots (Loh et al., 1993; Ho et al., 1994). An L-fucose-sensitive lectin produced by Bradyrhizobium sp. has also been shown to be involved in the attachment of this bacterium to the roots of its host plant, white lupin (Wisniewski \& Delmotte, 1996).

According to a model presented by Smit et al. (1987, 1992), an early step in the adhesion process is non-hostspecific. All members of the Rhizobiaceae utilize a common secreted calcium-binding protein, called rhicadhesin, to adhere to the root hairs of most plants. The rhicadhesin molecule is supposed to possess two binding domains, one for a receptor localized on the bacterial cell surface, and the other for a specific structure exposed on the root hair surface. The presence of $\mathrm{Ca}^{2+}$ ions was 
shown to be crucial in anchoring the protein to the cell surface of the bacteria (Smit et al., 1991). The direct binding mediated by rhicadhesin is followed by a step of clustering and firm attachment of the bacteria to the root hair surface, mediated by rhizobial cellulose fibres. Under manganese limitation also the plant lectin is involved in this interaction between bacteria and root hairs (Smit et al., 1987; Kijne et al., 1988). However, validation of this model is difficult since, to our knowledge, there are no reports describing the cloning of the gene for rhicadhesin or isolation of mutants defective in rhicadhesin activity.

Extracellular proteins, secreted by different proteinexport systems in different rhizobia, have attracted a great deal of interest during recent years. For example, it has been shown that culture media of $R$. leguminosarum contain several $\mathrm{Ca}^{2+}$-binding proteins, among them two exopolysaccharide glycanases, PlyA and PlyB, and the NodO protein. These are all secreted by a type I exporter system (Finnie et al., 1997, 1998). However, none of the secreted $\mathrm{Ca}^{2+}$-binding proteins has been recognized as rhicadhesin, although this protein was reported to be present in the culture medium of all members of Rhizobiaceae (Smit et al., 1989).

The phage-display technique, modified for cloning of genes encoding bacterial adhesins (Jacobsson \& Frykberg, 1996) is a powerful tool to identify and characterize interactions between bacterial and host proteins. As the name indicates, a peptide of interest is displayed on the surface of a filamentous phage by inserting DNA encoding the peptide into the phage genome. Since the peptide and its coding DNA are present in the same particle, it is possible to enrich for particles displaying an interacting peptide and then recover the coding DNA. Using this technique we tried to isolate the rhicadhesin gene utilizing the reported Rhizobium-binding property of the protein. This broad approach, which uses whole bacterial cells as the affinity substrate for phage enrichment, has the potential to reveal many interactions. We demonstrate the presence of several genes in the R. leguminosarum bv. trifolii genome that encode proteins able to bind to receptors exposed on the bacterial cell surface. One of these proteins, called RapA1, is characterized in more detail.

\section{METHODS}

Bacterial strains and growth media. The following Escherichia coli strains were used: TG1 as host in all experiments involving phages or phagemids; $\mathrm{DH} 5 \alpha$ in cloning experiments; MC1061 to express and purify the peptide encoded by the phage-display clone RA4; BL21(DE3)pLysS (Novagen), which enables stringent transcriptional control, to

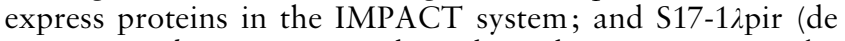
Lorenzo et al., 1990) to introduce plasmids into strain R200 by conjugation. Rhizobial strains used are listed below. R200 is a spontaneous streptomycin- and rifampin-resistant $\left(\mathrm{Sm}^{\mathrm{r}} \mathrm{Rif}^{\mathrm{r}}\right)$ mutant of Rhizobium leguminosarum bv. trifolii strain 2046 from the culture collection at the Department of Microbiology, Swedish University of Agricultural Sciences. Other strains (Sinorhizobium medicae USDA 1037, S. fredii USDA 257, S. teranga USDA 4894, $R$. leguminosarum bv. viciae
USDA 2370, R. leguminosarum bv. phaseoli USDA 2671, $R$. etli CFN42, R. tropici CIAT299, R. (Mesorhizobium) loti USDA $3471, R$. galegae USDA 4128, R. (Mesorbizobium) huakuii USDA 4779) were a kind gift of Antonio Lagares, University of La Plata, Argentina. Luria-Bertani liquid (LB) or solid (LA) medium supplemented with appropriate antibiotics was used to cultivate $E$. coli strains. TY ( 5 g tryptone, 3 g yeast extract per litre) or YMB $\left(0 \cdot 5 \mathrm{~g} \mathrm{~K}_{2} \mathrm{HPO}_{4}, 0 \cdot 1 \mathrm{~g} \mathrm{NaCl}, 10 \mathrm{~g}\right.$ mannitol, $0.4 \mathrm{~g}$ yeast extract and $0.2 \mathrm{~g} \mathrm{MgSO}_{4} \cdot 7 \mathrm{H}_{2} \mathrm{O}$ per litre) liquid or solid media with or without antibiotics were used for all Rhizobium strains. TY medium was supplemented with $\mathrm{CaCl}_{2}$ to final concentrations from $0.5 \mathrm{mM}$ to $7 \mathrm{mM}$. Antibiotics were used at the following concentrations: ampicillin (Amp) $50 \mu \mathrm{g} \mathrm{ml}^{-1}$, neomycin (Neo) $100 \mu \mathrm{g} \mathrm{ml}^{-1}$, chloramphenicol $(\mathrm{Cm}) 34 \mu \mathrm{g} \mathrm{ml}^{-1}$, rifampin (Rif) $25 \mu \mathrm{g} \mathrm{ml}^{-1}$ and streptomycin (Sm) $100 \mu \mathrm{g} \mathrm{ml}^{-1}$.

Construction of the phage-display library of $R$. leguminosarum bv. trifolii strain R200. The library was constructed in the pG8SAET phagemid vector (Jacobsson \& Frykberg, 1999) essentially as described by Jacobsson \& Frykberg (1995). In brief, genomic DNA of strain R200 was fragmented by sonication for $10-15 \mathrm{~s}$ into fragments with sizes between $0 \cdot 1$ and $5 \mathrm{~kb}$. The blunt-ended DNA fragments were ligated into the SnaBI-cleaved and calf intestinal phosphatase (CIP)treated vector using the Ready-to-go ligation kit (Amersham Pharmacia Biotech). The ligation mix was electroporated into electrocompetent TG1 cells yielding $9 \times 10^{7} \mathrm{Amp}^{\mathrm{r}}$ clones. Sixteen randomly picked clones all contained inserts with sizes between 0.1 and $2 \mathrm{~kb}$. Amplification of the clone library and preparation of the phage library was performed as in Jacobsson \& Frykberg (1995). The titre of phagemid particles in the final library was $10^{10}$ c.f.u. $\mathrm{ml}^{-1}$.

Selection of R200-binding phage from the library (pannings). Strain R200 was grown in TY medium supplemented with $1 \mathrm{mM} \mathrm{CaCl}$. Cells from $1 \mathrm{ml}$ of a late-exponential-phase culture were collected by centrifugation, washed once in an equal volume of TBS (Tris-buffered saline), resuspended in $0.3 \mathrm{ml} \mathrm{R} 200$ phagemid library supplemented with $2 \mathrm{mM} \mathrm{CaCl}_{2}$ and incubated for $2 \mathrm{~h}$ at room temperature on a shaker. Unbound phage were removed by washing the cells five times with $1.5 \mathrm{ml}$ TBS. After every wash the bacteria were gently spun down at 4000 r.p.m. for $3 \mathrm{~min}$ in a microfuge. After the last wash the cell pellet was mixed with $0.5 \mathrm{ml}$ of an E. coli TG1 culture to recover bound phage. This mixture was incubated for $30 \mathrm{~min}$ to allow the phage to infect the E. coli cells, and was subsequently plated on LA/Amp plates. Amp ${ }^{r}$ colonies were counted, washed off the plates, and an aliquot was superinfected with the helper phage to create an enriched library, which was used in the second enrichment cycle (repanning) according to the same protocol. After both cycles 150 colonies were picked and screened for E-tag expression using anti-E-tag antibodies (Amersham Pharmacia Biotech) and peroxidase-conjugated anti-mouse antibodies (Sigma). After the second cycle 16 E-tag positive colonies were chosen for sequence determination of the inserts.

DNA manipulations and construction of recombinant strains. Bluescript and pUC19 plasmids were used in cloning experiments. Plasmid pK18mob (Schäfer et al., 1994) was used to create vector insertion mutants of rapA1 and $r a p B$ genes in R200. PCR primers with introduced EcoRI sites were designed to amplify a $405 \mathrm{bp}$ internal part of the rapA1 gene. The generated PCR fragment was purified, cleaved with EcoRI and ligated into the EcoRI-cleaved pK18mob suicide vector. To create knock-out constructions for the $r a p B$ gene, the phagemid clone RA12 (Fig. 1) was cleaved with EcoRI. EcoRI cleaves out a fragment containing approximately $300 \mathrm{bp}$ of the 
insert and the gene VIII, encoding the major coat protein of the phage. This fragment was gel-purified and ligated into EcoRIcleaved pK18mob vector. After electroporation of both ligation mixtures into $E$. coli $\mathrm{DH} 5 \alpha$, clones with inserts in the sense and antisense orientations, relative to the orientation of $l a c Z^{\prime}$, were isolated. These plasmids were electroporated into E. coli strain S17-1 and introduced into strain R200 by mating. Recombinant transconjugants were selected on YMB agar plates containing Rif and Neo. Vector insertions in the desired loci were verified by PCR and sequencing. Recombinant strains R2014 and R2002 contain vector insertions in the rap $A 1$ gene in the sense and antisense orientation, respectively. Recombinant strain R2006 contains a vector insertion in the $r a p B$ gene in the sense orientation. The following strategy was used to obtain clones containing the unknown parts of the rapA1 and $r a p B$ genes and flanking regions. DNA was prepared from the recombinant strains R2014 and R2006, cleaved with HindIII, religated, and the ligation mixture was used to transform E. coli. Plasmids were prepared from $\mathrm{Neo}^{\mathrm{r}}$ transformants and cleaved with HindIII. Clones that yielded a single band after HindIII cleavage, indicating that no coligation of unrelated fragments or religation of partial cleavage fragments had occurred, were sequenced. A similar procedure was carried out with strain R2002 using the enzyme BamHI.

Plasmid and genomic DNA preparations, cloning, Southern blots, colony blots and hybridizations were carried out according to standard protocols (Sambrook et al., 1989). DNA was sequenced using the Thermo sequenase dye terminator cycle sequencing kit (Amersham) and an ABI PRISM 337 DNA Sequencer (Perkin Elmer) according to protocols provided by the manufacturers. Sequences were analysed with the Vector NTI Suite software package (InforMax, Inc.). Database searches were carried out using BLAST (Altschul et al., 1990).

Protein techniques and antibody preparation. A phagemid clone (RA4, Fig. 1) was chosen for purification of the peptide encoded by the insert, which was then used for production of anti-RapA antiserum. Rabbit anti-RapA-antiserum was prepared by Antibody AB, Södra Sandby, Sweden.

The RPAS Purification Module (Amersham Pharmacia Biotech) was used to purify the peptide fused to the E-tag. Preparation of cell lysates and protein purification were carried out according to the protocol provided with the kit.

For purification of anti-RapA antibodies from the antiserum, $1 \mathrm{mg}$ of the purified RapA1d protein (a derivative of RapA1, lacking 34 C-terminal amino acids) was coupled on a HiTrap affinity column (Amersham Pharmacia Biotech), and antibodies were purified from $4 \mathrm{ml}$ anti-RapA-antiserum according to the protocol supplied by the manufacturer. The resulting monospecific antibody preparation was adjusted to a concentration of $1 \mathrm{mg} \mathrm{ml}^{-1}$ and stored in the presence of $0.1 \mathrm{mg} \mathrm{ml}^{-1}$ BSA and $0.02 \% \mathrm{NaN}_{3}$ for later use in immunodetection assays.

The IMPACT T7 One Step Protein Purification System (New England Biolabs) was used to express and purify the whole RapA1 protein and RapA1d, following instructions provided by the manufacturer. The general protocol was adjusted to the given constructs as described below. Primers were designed to amplify the whole rapA1 gene, or part of it, by PCR using R200 genomic DNA as template. NcoI and SmaI sites were introduced in the forward and reverse primers, respectively, to allow cloning into the pTYB4 vector, supplied with the kit. Clones were tested for PCR-induced sequence errors by sequencing. Cells were lysed by repeated cycles of freezing and thawing.
Proteins were analysed by SDS-PAGE using the Phast-system (Amersham Pharmacia Biotech) with PhastGel Gradient $8-25 \%$ and PhastGel SDS Buffer Strips. Proteins were blotted onto nitrocellulose filters by capillary action. In Western blot analysis the filters were incubated with appropriate primary antibodies and secondary peroxidase-labelled antibodies. Bound antibodies were detected using 4-chloro-1-naphthol.

Immunofluorescence microscopy. R200 cells from $1 \mathrm{ml}$ TY or YMB cultures of various ages were collected, washed once with TBS (Tris-buffered saline) and incubated with antiRapA-antibodies, diluted $1: 100$ in TBS containing $0.5 \mathrm{mg} \mathrm{ml}^{-1}$ BSA. After three washes with TBS, the cells were incubated with FITC-conjugated goat anti-rabbit antibody preparation (diluted $1: 160$ in TBS containing $0.5 \mathrm{mg} \mathrm{BSA} \mathrm{ml}^{-1}$ ). After three washes with $1 \mathrm{ml}$ TBS, an aliquot of the cell suspension was mounted on a microscope slide, covered with a cover slip and observed in a Zeiss axioscope with a filter set containing exciter filter BP 450-490, chromatic beam splitter FT510 and barrier filter LP520.

In some experiments cells were preincubated with RapA1 and RapA1d proteins or BSA $\left(100 \mu \mathrm{g} \mathrm{m}^{-1}\right)$ in the presence of $2 \mathrm{mM} \mathrm{CaCl}_{2}$ and $0.5 \mathrm{mg} \mathrm{BSA} \mathrm{m}^{-1}$, and washed before adding the antibodies. All incubations were carried out for $1 \mathrm{~h}$ at room temperature on a shaker. Cells were collected by gentle centrifugation at 4000 r.p.m. for $4 \mathrm{~min}$ throughout the experiment.

Particle agglutination assay (PAA). Latex beads (Standard Dow Latex, Serva) $0.552 \mu \mathrm{m}$ in diameter were used and the assay was performed essentially as described by Styriak et al. (1999). Purified RapA1 protein $(100 \mu \mathrm{g})$ or BSA $(1000 \mu \mathrm{g})$ was used to coat aliquots of $200 \mu \mathrm{l}$ beads. For the PAA, bacteria from different cultures were washed and suspended in TBS to approximately $10^{10}$ c.f.u. $\mathrm{ml}^{-1}$. Equal volumes of latex beads in TBS and bacterial suspensions were mixed on glass slides and agglutination was observed visually. Clearly visible agglutination of the particles appearing within $5 \mathrm{~min}$ after mixing the beads and the bacteria was scored as a positive reaction, indicating an interaction between bacteria and the protein coated on the beads.

${ }^{45} \mathrm{Ca}^{2+}$-binding assay. An SDS-PAGE gel carrying $\sim 6 \mu \mathrm{g}$ recombinant RapA1 protein, the same amount of RapA1d protein and protein molecular mass standards was electroblotted to a nitrocellulose filter and assayed for the presence of $\mathrm{Ca}^{2+}$-binding proteins as described by Maruyama et al. (1984) using ${ }^{45} \mathrm{Ca}^{2+}$ (Amersham Pharmacia Biotech). The filter was exposed to Kodak Biomax MR film for $3 \mathrm{~d}$.

Precipitation of proteins and EPS from culture media. Proteins were precipitated from different culture media with trichloracetic acid (TCA). TCA was added to sterile-filtered culture media on ice to a final concentration of $5 \%$. Precipitated proteins were collected by centrifugation at 14000 r.p.m. in a microfuge, washed with acetone, dried and dissolved in water.

A crude exopolysaccharide (EPS) preparation was obtained by precipitating EPS from sterile-filtered supernatant of a 5-d-old R200 culture in TY medium with 3 vols ethanol. The precipitate was dried and dissolved in water.

\section{RESULTS}

\section{Phage-display cloning reveals a family of homologous cell-surface-binding proteins}

Rhicadhesin is, according to Smit et al. (1989), a universal rhizobial adhesin proposed to bridge the distance between bacteria and root hair cells by binding 
with one domain to a bacterial receptor and with another domain to a root-borne receptor. A decrease in the $\mathrm{Ca}^{2+}$ concentration of the growth medium renders the bacteria unable to bind secreted rhicadhesin, which is then mainly found in the growth medium, leaving the receptors on the bacterial cell surface free (Smit et al., 1991). The rationale of our approach was that such bacteria could serve as a suitable substrate for selection of rhicadhesin-expressing phage from a rhizobial phagedisplay library.

A phage-display library of R. leguminosarum bv. trifolii strain R200 was constructed as described in Methods. For the enrichment procedure (biopannings), strain R200 was cultivated to late exponential phase in TY medium supplemented with $1 \mathrm{mM} \mathrm{CaCl}$. Lowering the $\mathrm{Ca}^{2+}$ concentration of the TY medium under $1 \mathrm{mM}$ abolished growth. The bacteria were harvested, washed and used to enrich for Rhizobium-adhering (RA) phage from the library. Previous experience had shown (Jacobsson \& Frykberg, 1999) that two panning cycles are often sufficient to obtain a specific enrichment of up to $100 \%$ for clones encoding the desired interaction. Monitoring of E-tag expression (a specific short tag sequence, expressed only when the insert is fused in the correct reading frame to the sequence encoding the phage coat protein) allows a preliminary estimation of the enrichment frequency. After the first panning, the number of recovered phagemids was approximately $10^{4}$, of which $23 \%$ expressed the E-tag (the occurrence of Etag-positive clones in the unselected library is $1-2 \%$ ). Repanning yielded $10^{6}$ phagemids, of which $79 \%$ were E-tag positive. Both features, the increase in the total number of recovered phagemids and the increase in the number of E-tag-positive clones, indicated that specific enrichment had taken place. Sixteen E-tag-positive clones from the second cycle were chosen for sequence determination of the inserts. Sequence analysis revealed several types of overlapping inserts, which were divided into three main groups as shown schematically in Fig. 1.

Overlapping inserts of clones RA1-8 (group A, subgroup A.1; Fig. 1) contained the $5^{\prime}$-part of an open reading frame and parts of an upstream sequence. The overlapping clones RA9 and RA10 (group A, subgroup A.2; Fig. 1) contained a very similar sequence, with a few disperse mutations in the coding region of the inserts, as compared to the A.1 sequence. However, the upstream sequences of the two subgroups were different, suggesting the presence of two related genes at different genomic locations. These genes were designated rapA1 and rap $A 2$. The distribution of inserts of different lengths from rapA1 and rapA2 allowed estimation of the minimal sequence required for binding to the R200 cell surface. This sequence, called the $\mathrm{Ra}$ (Rhizobiumadhering) domain, is present in all clones in the group as shown in Fig. 1. The overlapping clones RA11 and RA12 (group B; Fig. 1) were also found to encode part of a protein that contains a motif homologous to the $\mathrm{Ra}$ domain, whereas the sequences outside the conserved motif were different from those in group A. The corresponding gene was named rapB. Also the insert of clone RA13 (group C; Fig. 1) coded for a peptide with homology to the Ra domain, but its sequence was not identical to those in the other two groups. We therefore propose that this peptide represents a third type of Rap protein, here denoted RapC. Clones 14-16 contained very short (less than $100 \mathrm{bp}$ ) inserts and were not further investigated.

\section{One member of the Rap protein family, RapA1, consists of two homologous repeats, which display sequence similarity to the PlyA protein in $R$. leguminosarum}

Phage-display cloning generally reveals only the binding domain of a protein. Even in cases where the phagemid clones span most of the gene sequence, the very 3 '-end of the gene will be absent, as the method does not allow a stop codon between the insert and gene VIII of the phage. We decided to clone the complete rapA1 gene, as being most abundantly represented among the RA phagemids, and the rapB gene, as being most different from the other recognized genes.

A probe created from a rapA1-containing phage-display clone was used to hybridize a Southern blot of R200 genomic DNA cleaved with different enzymes. Multiple bands were observed in all lanes, in agreement with a tentative redundant gene family. Two methods were used to obtain the complete sequence of the rapA1 and rap $B$ genes. First, an approximately $2 \mathrm{~kb}$ fragment containing the rapA1 sequence was cloned, using the Southern hybridization data, and partially sequenced. However, difficulties encountered using this strategy indicated that the gene product might be toxic to E. coli cells. To avoid this problem, we generated mutant strains with vector insertions in the rapA1 or the rapB genes. Releasing the vector from these strains with different restriction enzymes provided clones that contained parts of the genes together with flanking sequences of different lengths. Using this method, $2017 \mathrm{bp}$ of rapA1 sequence was obtained (GenBank accession number AF265222). An open reading frame of $696 \mathrm{bp}$, representing the rapA1 gene, was detected in that sequence. The predicted RapA1 protein is 232 aa long and consists of two homologous repeats of similar length. The size of each repeat corresponds approximately to the size of the Ra domain (Fig. 1), the minimal sequence needed for the Rhizobium-binding property. This suggests that one repeat constitutes one receptorbinding site. Hence, the presence of two homologous repeats in the RapA1 protein would result in divalency in receptor binding. The sequence from 890 bp downstream of the rapA1 stop codon shows $100 \%$ identity to a sequence encoding PssP, an EPS polymerization protein in R. leguminosarum bv. trifolii (J. E. Krol and others, unpublished; GenBank AF067140) as well as extensive similarity to a sequence encoding ExoP, a succinoglycan-transport protein in $S$. meliloti (Glucksmann et al., 1993). This indicates that rapA1 is located near a cluster of genes involved in the synthesis of acidic EPSs, symbiotically active substances in several rhizobia. 


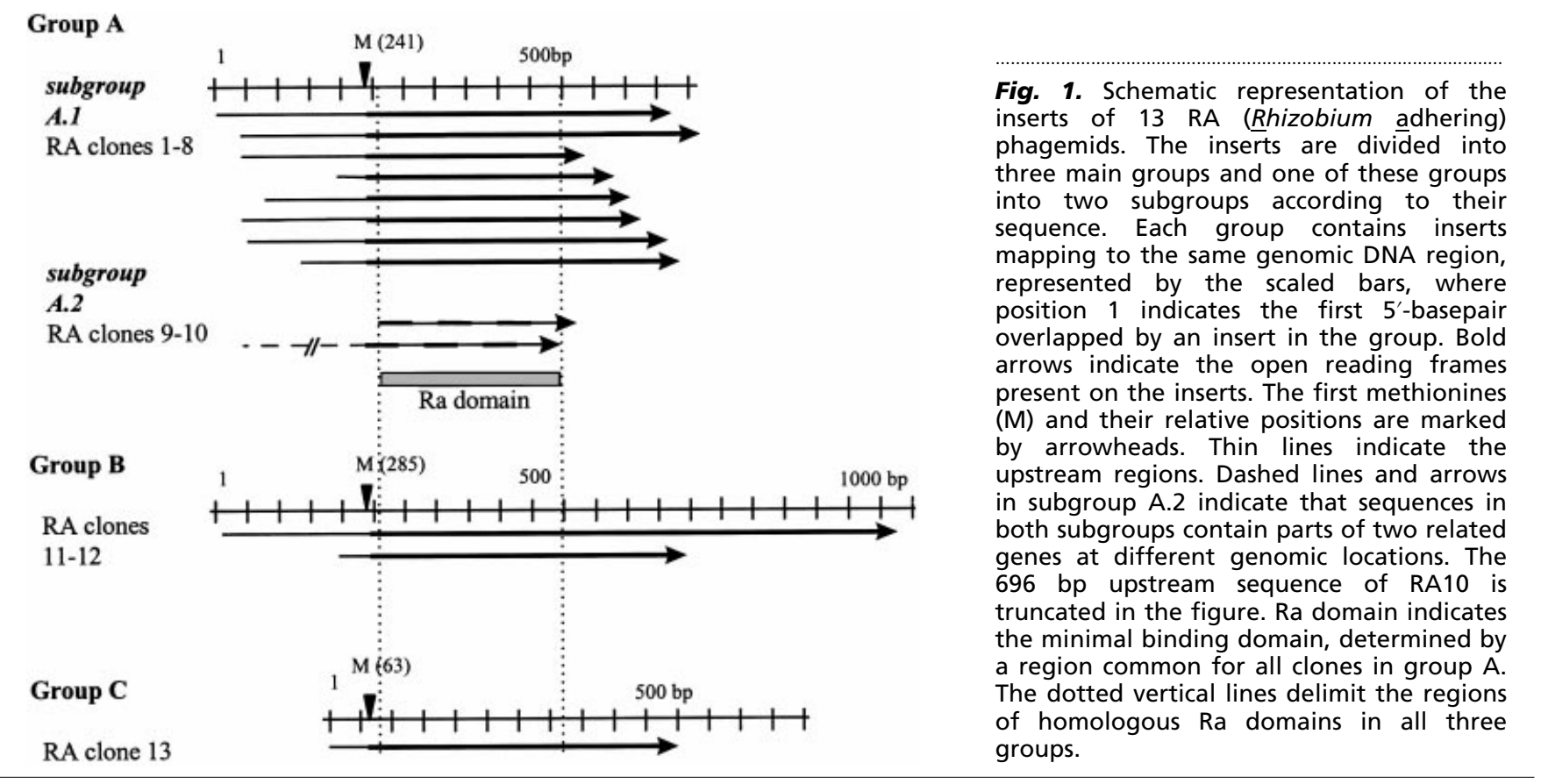

The complete sequence of the $\operatorname{rap} B$ gene showed that it encodes a putative protein of 279 aa (GenBank accession number AF265223). Interestingly, there was only one Ra domain in this protein, located in the $\mathrm{N}$-terminal part, while the rest of the sequence did not show homology to RapA1. However, limited sequence similarity between the C-terminal half of RapB and an endoglucanase (alkaline cellulase) from Bacillus sp. was found in a BLAST search. The sequence downstream of the rapB gene shows high similarity to the $y 4 l L$ gene located on the symbiotic plasmid of Rhizobium sp. NGR234

The partial sequence of the rapC gene (GenBank accession number AF315810), derived from clone RA13 (Fig. 1), contains one entire $\mathrm{Ra}$ domain and the beginning of a second homologous $\mathrm{Ra}$ domain, resembling the structure of the rapA1 gene. It also shows higher sequence homology to the rapA1 and rapA2 (GenBank accession number AF315809) genes than to the $\operatorname{rapB}$ gene (Fig. 2).

The homologous Ra domains of RapA1, RapA2, RapB and RapC proteins (Fig. 1) showed homology also to the C-terminal part of the PlyA protein from R. leguminosarum (Finnie et al., 1998). This was the only protein with a known function in the databases that exhibited sequence similarity to the Rap proteins according to the standard BLAST search. Alignment of the Rhizobiumbinding domains of the RapA, B and C proteins and the C-terminal part of the PlyA protein is shown in Fig. 2. Finnie et al. (1998) described two homologous glycanases PlyA and PlyB, which degrade EPS and carboxymethylcellulose (CMC). They display sequence similarity to several polysaccharide lyases. The Cterminal parts of the PlyA and PlyB proteins (amino acids 419-540 in PlyA and 396-497 in PlyB), outside the region of similarity to other glycanases, apparently contain an Ra domain (Fig. 2). The putative function of the Ra domain in PlyA and PlyB is discussed later.

\section{RapA1 is a secreted protein found in all $R$. leguminosarum biovars and $R$. etli}

The results presented above showed that we have identified several proteins that are able to interact with the bacterial cell surface, although the predicted sizes of the RapA1 and RapB proteins $(24$ and $30 \mathrm{kDa}$, respectively) do not agree with the reported size of rhicadhesin $(14 \mathrm{kDa})$. Our next aim was to find out whether RapA1 is a true extracellular protein. Affinitypurified anti-RapA antibodies were used as a specific tool to study the expression of the RapA proteins in different rhizobia. We refer to RapA proteins, since the immunodetection with anti-RapA antibodies does not distinguish between RapA1 and RapA2. We also expressed and purified the recombinant proteins RapA1 and RapA1d (a truncated derivative of RapA1, lacking $34 \mathrm{aa}$ in the C-terminus) using the IMPACT system, which gives a recombinant protein without affinity tag.

Proteins were precipitated from sterile-filtered growth media of 11 Rhizobium species and assayed for the presence of RapA proteins by SDS-PAGE and subsequent Western blot analysis. The strains tested were R. leguminosarum bv. trifolii strain R200, R. leguminosarum bv. viciae USDA 2370, $R$. leguminosarum bv. phaseoli USDA 2671, S. medicae USDA 1037, S. fredii USDA 257, $S$. teranga USDA 4894, $R$. etli CFN42, $R$. tropici CIAT299, $R$. loti USDA 3471, $R$. galegae USDA 4128 and $R$. huakuii USDA 4779. Only culture medium preparations from $R$. leguminosarum bvs trifolii, viciae and phaseoli, and R. etli, were found to contain proteins 
(a)

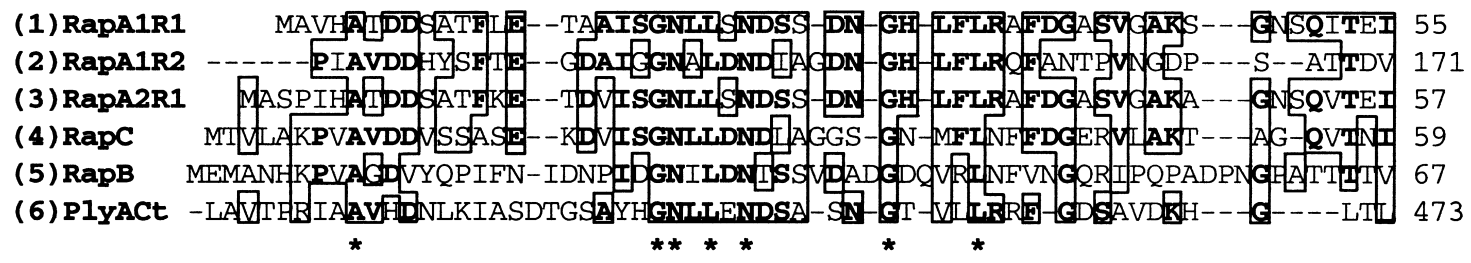

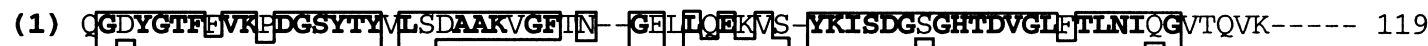

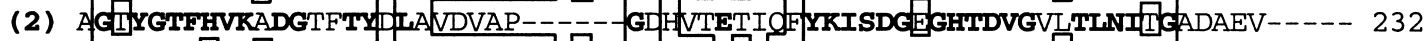

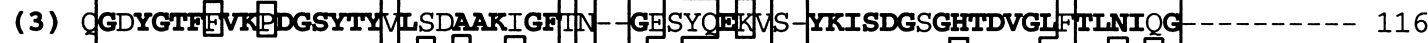

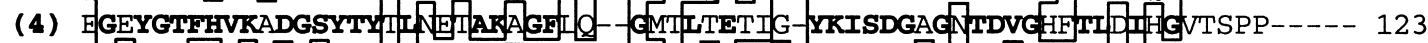

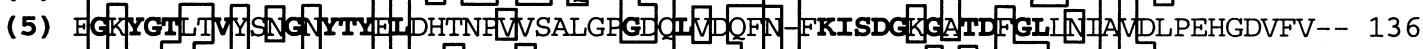

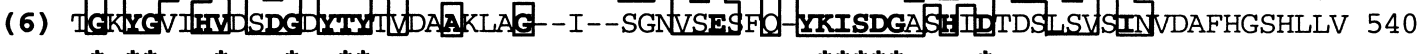

(b)

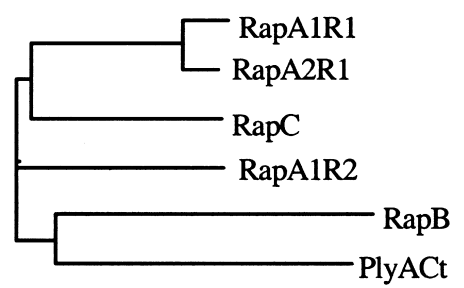

Fig. 2. CLUSTAL alignment (a) and the phylogenetic tree (b) of different Rhizobium adhering (Ra) domains of RapA1, RapA2, RapB, RapC and PlyA proteins. RapA1R1 and RapA1R2 refer to the first and the second repeat structure in the RapA1 protein. RapA2R1 refers to the first repeat in the RapA2 protein. PlyACt refers to the C-terminal part of the PlyA protein containing an Ra domain. Amino acid positions refer to locations in the whole proteins. Blocks containing similar, conservative or identical amino acids are boxed. Conserved residues are in bold. Amino acids conserved in all six sequences are indicated $\left(^{*}\right)$ beneath the alignment. Alignment and the phylogenic tree were calculated using the Vector NTI Suite software package with default settings.

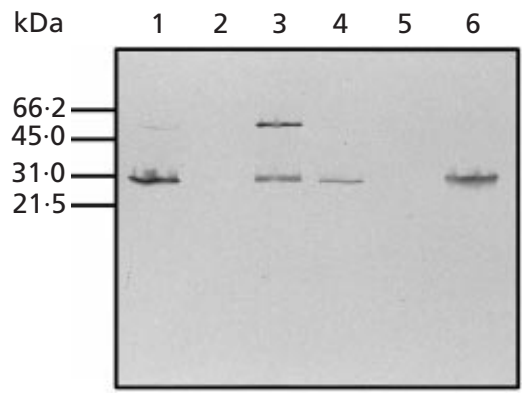

Fig. 3. Presence of RapA proteins in the culture medium of different rhizobia. A Western blot filter containing precipitated proteins from growth media of $R$. leguminosarum bv. trifolii R200 (lane1), S. fredii USDA257 (lane 2), R. leguminosarum bv. phaseoli USDA2671 (lane 3), $R$. etli CFN42 (lane 4), $R$. tropici CIAT299 (lane5) and $R$. leguminosarum bv. viciae USDA2370 (lane 6) was probed with anti-RapA antibodies and secondary peroxidase-labelled antibodies.

that reacted with anti-RapA-antibodies (Fig. 3). The bands on the Western blot filter around $30 \mathrm{kDa}$ were in agreement with the size of the recombinant RapA1 protein (data not shown). The extra bands in lanes 1 and 3 (Fig. 3) probably represent RapA-related proteins of higher molecular mass in these species. The corresponding bands on Coomassie blue-stained gels were very faint, suggesting that RapA proteins are not abundant among the secreted proteins (data not shown). SDS-PAGE and Western blots were also performed with lysates of washed cells from cultures of strain R200 at different growth stages. None of these cell lysate preparations reacted with anti-RapA antibodies (data not shown), indicating that at least the major fraction of the protein was extracellular and not cell-surfaceassociated.

\section{RapA1 is a calcium-binding protein}

Culture medium of $R$. leguminosarum bv. viciae has been shown to contain several calcium-binding proteins, some of which are secreted via the PrsDE type I exporter (Finnie et al., 1997, 1998). In this paper we demonstrate that culture medium from $R$. leguminosarum bv. viciae also contains a RapA protein (Fig. 3, lane 6). The $\mathrm{Ca}^{2+}$ binding properties of the recombinant proteins RapA1 and RapA1d, and protein size markers, separated on 
(a)

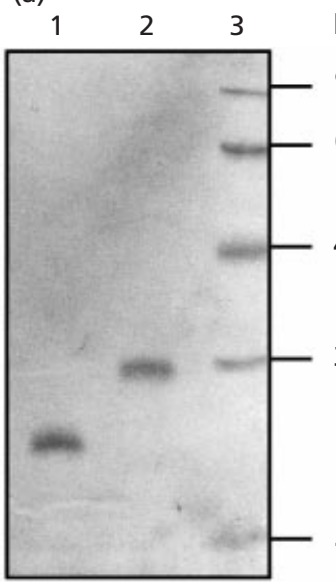

(b)

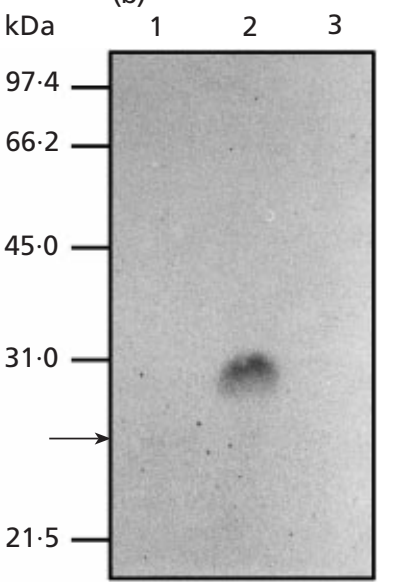

Fig. 4. $\mathrm{Ca}^{2+}$-binding property of the RapA1 protein. (a) Coomassie-blue-stained SDS-PAGE gel, containing RapA1d (lane 1), RapA1 (lane 2) and protein size markers (lane 3). (b) Autoradiograph of a filter containing the same proteins as in (a), electroblotted from a duplicate gel and incubated with ${ }^{45} \mathrm{Ca}^{2+}$. The arrow indicates the position of a very weak band corresponding to the RapA1d protein.

SDS-PAGE and electroblotted onto a nitrocellulose filter, were assayed with ${ }^{45} \mathrm{Ca}^{2+}$. The results presented in Fig. 4 clearly show that RapA1 binds $\mathrm{Ca}^{2+}$. Although equal amounts of both proteins were loaded on the gel, the full-length RapA1 gave a significantly stronger signal compared with the truncated RapA1d protein. This indicates that the $34 \mathrm{C}$-terminal amino acids, which are lacking in RapA1d, are involved in binding of $\mathrm{Ca}^{2+}$.

In most proteins secreted via the type I secretion system, a $\beta$-roll structural element, composed of several glycineand aspartate-rich nonapeptide repeats, is believed to be responsible for $\mathrm{Ca}^{2+}$ binding (Lilie et al., 2000). The sequence of the PlyA protein also contains a repetitive motif, consisting of 10 copies of a heptapeptide repeat (Finnie et al., 1998). No obvious repeat region of similar kind is present in the RapA1 sequence, although the protein is rich in glutamate and aspartate residues, which are potential ligands for $\mathrm{Ca}^{2+}$ ions.

\section{RapA1 is a polar cell-surface-associated agglutinin}

To visualize the Rhizobium-adhering property and the cell-surface location of the RapA1 protein, an indirect immunofluorescence assay with intact R200 cells was performed. Cells from a late-exponential-phase culture in TY medium supplemented with $0.1 \% \mathrm{CaCl}_{2}$ were divided into three samples. Sample (a) was incubated with BSA, sample (b) with recombinant protein RapA1d (a derivative of RapA1, lacking 34 C-terminal amino acids), and sample (c) with full-length recombinant protein RapA1. All three samples were then treated with anti-RapA-antibodies and FITC-labelled secondary antibodies, and then observed under a fluorescence micro-
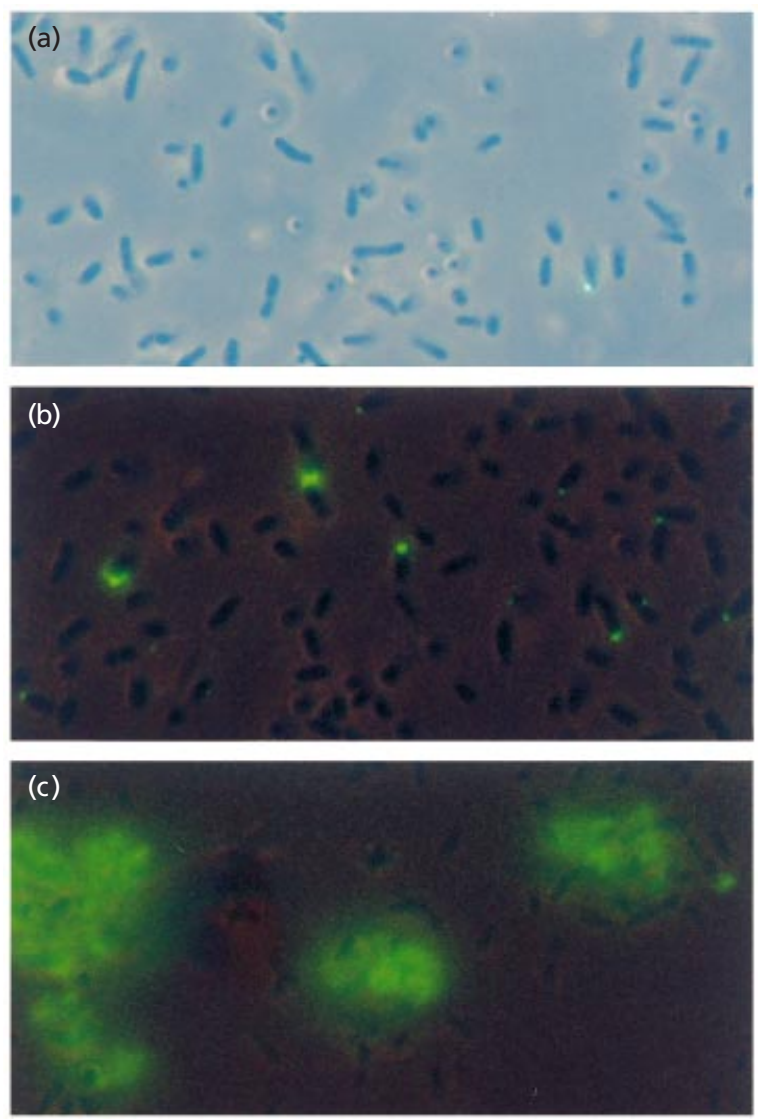

Fig. 5. Immunofluorescence of $R$. leguminosarum bv. trifolii strain R200. Bacteria were harvested from an exponential-phase TY culture and incubated with BSA (a) and with RapA1d (b) and RapA1 (c) proteins, followed by treatments with anti-RapAantibodies and secondary FITC-labelled antibodies.

scope (Fig. 5). In sample (a) less than $0 \cdot 1 \%$ of cells showed a fluorescent signal (Fig. 5a). This agrees with our observation (see above) that the cell lysate preparations did not react with anti-RapA antibodies. All observed signals were located exclusively at one pole of the bacteria. In sample (b), incubated with RapA1d, $10-20 \%$ of the bacterial cells showed a unipolar signal (Fig. 5b). This indicates that during the exponential phase of growth the bacteria express receptor molecules that are free for binding added RapA1 protein. We also conclude that the receptor for the first $\mathrm{Ra}$ repeat is located predominantly at one pole of the bacteria. Sample (c), in which the full-length RapA1 protein was used, contained big, strongly fluorescing clumps of aggregated bacteria (Fig. 5c), showing that the RapA1 protein, containing two functional $\mathrm{Ra}$ domains, is able to agglutinate the bacteria. The inability of the RapA1d protein to promote aggregation indicates that deletion of 34 amino acids from the second $\mathrm{Ra}$ domain renders it non-functional. This also supports the interpretation that one entire $\mathrm{Ra}$ domain constitutes one receptor binding site. 

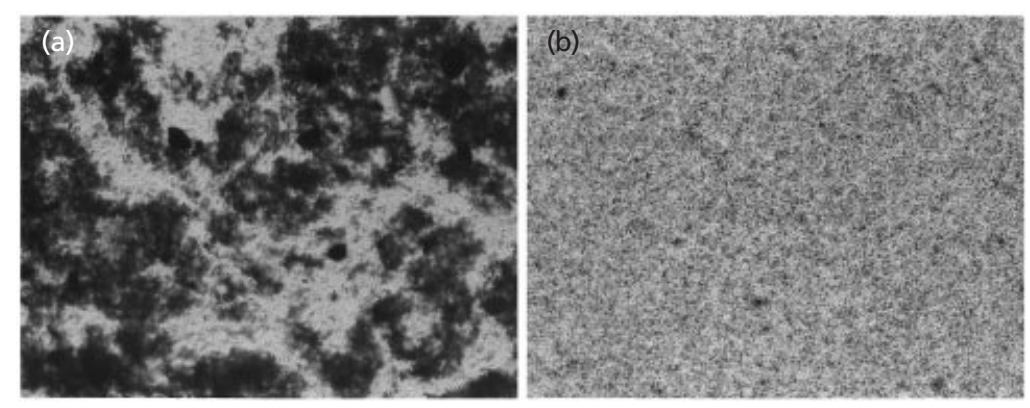

Fig. 6. Particle agglutination assay. Exponential-phase R200 cells were mixed with RapA1-coated latex beads (a) or BSAcoated beads (b) on a microscope slide. Agglutination was recorded under a phasecontrast microscope at $100 \times$ magnification 5 min after mixing of bacteria and beads.

\section{The cell-surface receptor for RapA1 is produced only by the same group of rhizobia that express the RapA proteins}

In order to study the specificity of the agglutination and factors influencing it, a particle agglutination assay (PAA) was used. Small latex beads of approximately the same size as bacteria were coated with recombinant RapA1 protein. Control beads were coated with BSA. RapA1-coated beads strongly agglutinated exponentialphase R200 cells, while the mixture of BSA-coated beads and R200 cells remained homogeneous (Fig. 6), showing that the PAA can be used as an assay system to study the specificity of the interaction between RapA1 and bacteria. No aggregation was observed when RapA1-coated beads were mixed with E. coli cells. R200 cells from exponential-phase TY cultures always showed faster and stronger responses in the PAA assay, compared to cells in stationary phase, indicating the presence of a maximal amount of free cell-surface receptors for RapA1 protein during exponential phase. Agglutination was not affected by the $\mathrm{Ca}^{2+}$ concentration of the growth medium. Preincubation with $2 \mathrm{mM} \mathrm{Ca}^{2+}$ enhanced the agglutinating activity of the RapA1-coated beads, indicating that $\mathrm{Ca}^{2+}$ ions bound to the protein promote the binding of RapA1 to its receptor. A slow and weak agglutination also occurred without added $\mathrm{CaCl}_{2}$, which may be explained by the presence of $\mathrm{Ca}^{2+}$ bound to the bacteria during growth.

We also tested the ability of the RapA1 protein to agglutinate different rhizobia. The same set of strains, previously assayed for the presence of the RapA proteins, was used in the PAA. Only cells of $R$. leguminosarum biovars trifolii, phaseoli and viciae, and $R$. etli, agglutinated the RapA1-coated beads (data not shown). These strains had earlier been shown to express RapA proteins (see Fig. 3). Hence the production of the receptor is a property specific only for strains that express the RapA proteins.

The rapA1 gene is located near a gene cluster involved in EPS production, suggesting that the RapA1 receptor might be a carbohydrate structure. Aliquots of RapA1coated beads were incubated with a crude EPS preparation from strain R200, washed and then tested in the PAA with R200 cells. This treatment abolished the agglutinating activity of the RapA1-coated beads, suggesting that the crude EPS preparation contains molecules that are compatible with the RapA1 cell- surface receptors. The nature of these molecules will be studied in the future.

\section{DISCUSSION}

\section{The Rap proteins are different from rhicadhesin}

The purpose of this investigation was to isolate the gene for rhicadhesin, a rhizobial attachment protein described by Smit et al. (1989), by using phage-display cloning. Our approach was based on using intact $R$. leguminosarum bv. trifolii cells as the affinity substrate for enrichment of phage expressing rhicadhesin, from a phage-display library constructed from strain R200. Since the bacterial cell surface is a complex structure, one could expect to find several interacting proteins, rhicadhesin being one of them. Four proteins able to interact with the bacterial cell surface were found, here designated Rhizobium-adhering proteins RapA1, RapA2, RapB and RapC. Interestingly, the RapA1 protein, characterized in more detail in the present paper, has several striking similarities with rhicadhesin. It is a secreted calcium-binding protein (Figs 3 and 4), which can bind to a receptor on the rhizobial cell (Figs 5 and 6). Also similar to rhicadhesin, RapA1 has two different binding domains and is able to form a bridging structure. However, in contrast to rhicadhesin, RapA1 is produced only by a small group of rhizobia, $R$. leguminosarum biovars and R. etli (Fig. 3). Also the predicted molecular masses of all Rap proteins are higher than $14 \mathrm{kDa}$, which was the reported size for rhicadhesin (Smit et al., 1989). A possible reason why the gene for rhicadhesin was not revealed might be that rhicadhesin-expressing phage were outcompeted by Rap-expressing phage in the pannings.

\section{Ra domains are found as building blocks in different proteins}

No predictions concerning the functions of the Rap proteins could be derived from sequence homologies to proteins in the databases. The PlyA protein from $R$. leguminosarum bv. viciae was the only protein with a known function that showed sequence similarity to $\mathrm{Ra}$ proteins. This similarity is limited to a short C-terminal part in PlyA (Fig. 2) and also in PlyB. Furthermore, PlyA and PlyB have catalytic domains responsible for degrading EPS and CMC, and nonapeptide repeat regions, 
supposed to be involved in the binding of $\mathrm{Ca}^{2+}$ (Finnie et al., 1998). The sequence of RapA1 does not contain any of these features, but consists of two homologous repeats (Ra domains). PlyA and PlyB have been shown to be able to cleave EPS and CMC only when activated by an EPS-related structure (Zorreguieta et al., 2000). This, together with our data showing that each $\mathrm{Ra}$ domain constitutes a receptor-binding site, makes it tempting to speculate that this domain in PlyA and PlyB might be responsible for binding to a specific EPS structure, resulting in activation of the enzymic activity of these proteins. In this respect it is of interest that the activation of PlyA and PlyB was observed only with EPS from $R$. leguminosarum biovars and $R$. etli, the same group of rhizobia found to secrete the RapA1 protein and to express the cell-surface receptor for that protein, as described in this paper.

Compared to PlyA, the Ra domain has a different position in the RapB protein, where it constitutes the $\mathrm{N}$ terminal half of the protein. Interestingly, although the RapA1 and RapB proteins have similar molecular masses, amino acid compositions and isoelectric points, the sequence of the C-terminal half of RapB does not show any homology to the R domain sequence. Instead, it exhibits weak sequence similarity to an endoglycanase from Bacillus. Thus it seems that the Ra domain is an independent functional unit which can be integrated into various locations in different proteins, e.g. Cterminally in PlyA and N-terminally in RapB.

\section{Is RapA1 a lectin?}

There are several reasons to speculate that receptors for Rap proteins are carbohydrate structures : the proximity of the rapA1 gene to the gene cluster involved in EPS synthesis; sequence similarity between the Rap proteins and PlyA and PlyB, involved in processing EPS; and the ability of the crude EPS preparation to inhibit the binding of RapA1 to its cell-surface receptors. Carbohydrate-binding proteins that specifically recognize diverse sugar structures are generally referred to as lectins. In some respects, RapA1 resembles the bradyrhizobial BJ38 lectin (Ho et al., 1994). Both proteins are polarly cell-surface-associated and are able to agglutinate the bacteria. The formation of aggregates containing polarly bound bacteria (star formation) was shown to be BJ38 dependent (Ho et al., 1990). We have demonstrated that the recombinant RapA1 protein agglutinates R 200 cells (Fig. 6c). BJ38 has also been shown to be involved in the binding of B. japonicum to soybean roots (Ho et al., 1994). We have also observed, using immunofluorescence assay, that the recombinant RapA1 protein has affinity to the root hair surface (data not shown). However, the specificity of this interaction is not clear, since the root hair tip appears to be a highly adsorptive surface. For example, both BSA- and RapA1-coated latex beads readily adhered to the tip region of young root hairs, whereas the same BSA-coated beads did not show any background activity in the particle agglutination assay with whole R200 cells.

\section{The transient expression of RapA1 receptors is another example of polar growth-phase-dependent structures exposed on the cell surface of $R$. leguminosarum bv. trifolii}

Bacteria in the exponential phase had the highest capability to bind recombinant RapA1 protein in the PAA. The reduced agglutination when bacteria from the stationary phase were used could be explained by saturation of the receptor sites with extracellular RapA proteins. However, bacteria in the stationary phase did not show a higher percentage of fluorescing signals under the microscope compared with exponential-phase bacteria. Furthermore, lysates of both stationary and exponential-phase cells did not react with anti-RapA antibodies in Western blots. This means that the functional cell-surface receptors of the RapA proteins are present mainly in the exponential growth phase and that the expression of the proteins is low at any growth phase. This correlates with the concept of the rhizobial cell surface as a dynamic structure which undergoes changes during growth. Transient appearance of plant lectin receptors on the bacterial cell surface has been demonstrated for B. japonicum and R. leguminosarum bv. trifolii (Bhuvaneswari et al., 1977, 1983; Dazzo et al., 1979; Hrabak et al., 1981). Development of the capsule and the lectin receptors in both species has been shown to proceed through several phases, starting from one pole of the bacteria (Sherwood et al., 1984; Vasse et al., 1984). We have shown that, in addition to transient appearance, the cell-surface receptors of RapA1 are located at one pole of the bacteria (Fig. 6a, b). Development of such polarity has often been suggested to have consequences in the symbiotic process. The attachment of rhizobia to root hair surfaces often occurs in a polar way. The polar attachment of $R$. leguminosarum bv. trifolii to clover root hairs is favoured by the polar alteration of the capsule by enzymes in the clover root exudate and also by the age-dependent distribution of lectin receptors on the bacterial cell surface (Dazzo et al., 1982; Sherwood et al., 1984). The pole carrying the trifoliin A receptors has been reported to make direct contact with root hairs (Dazzo et al., 1984). Polarity is a well-characterized feature also in the attachment behaviour of B. japonicum, where the BJ38 lectin and the receptors for the soybean lectin are situated at opposite poles. The BJ38 pole has been shown to make the contact with the soybean root (Loh et al., 1993). At present we do not know if the RapA pole corresponds to the trifoliin A pole (Dazzo et al., 1982; Sherwood et al., 1984) or the opposite one. If the latter is true, it would imply a similar organization of adhesive molecules on the cell surface of both species.

\section{Conclusions}

In this paper we show that $R$. leguminosarum bv. trifolii secretes an agglutinin, called RapA1, which recognizes receptors on its own surface. The receptors are expressed during the exponential phase and their presence results in agglutination of the bacteria when purified recom- 
binant RapA1 protein is added. However, no significant aggregation occurs during ordinary in vitro growth, which agrees with the observed low expression of the protein under these conditions. We speculate that under in vivo conditions environmental factors influence its expression level. The presence of two related rapA genes (rapA1 and rapA2) with different upstream regions might allow differential expression of the agglutinin under different conditions. Future research will be directed to clarification of the chemical nature of the receptors of the Rap proteins. It is possible that the Rap proteins are lectins, which - similar to trifoliin A or soybean lectin - recognize sugar structures present in both plants and bacteria. This would support our hypothesis that the RapA1 protein has a role in the attachment process similar to the BJ38 lectin in $B$. japonicum.

\section{ACKNOWLEDGEMENTS}

This work was supported by a grant from the Swedish Council for Forestry and Agricultural Research (32.0412/98) and Carl Trygger's Foundation for Scientific Research to M.L.

We thank Professor Gerhart Wagner for critical reading of the manuscript.

\section{REFERENCES}

Altschul, S. F., Gish, W., Miller, W., Myers, E. W. \& Lipman, D. J. (1990). Basic local alignment tool. J Mol Biol 215, 403-410.

Bhuvaneswari, T. V., Pueppke, S. G. \& Bauer, W. D. (1977). Role of lectins in plant-microorganism interactions. I. Binding of soybean lectin to rhizobia. Plant Physiol 60, 486-491.

Bhuvaneswari, T. V., Mills, K. K., Crist, D. K., Evans, W. R. \& Bauer, W. D. (1983). Effects of culture age on symbiotic infectivity of Rhizobium japonicum. J Bacteriol 153, 443-451.

Dazzo, F. B. \& Hubbell, D. H. (1975). Cross-reactive antigens and lectin as determinants of symbiotic specificity in the Rhizobiumclover association. Appl Microbiol 30, 1017-1033.

Dazzo, F. B., Urbano, M. R. \& Brill, W. J. (1979). Transient appearance of lectin receptors on Rhizobium trifolii. Curr Microbiol 2, 15-20.

Dazzo, F. B., Truchet, G. L., Sherwood, J. E., Hrabak, E. M. \& Gardiol, A. E. (1982). Alteration of the trifoliin A-binding capsule of Rhizobium trifolii 0403 by enzymes released from clover roots. Appl Environ Microbiol 44, 478-490.

Dazzo, F. B., Truchet, G. L., Sherwood, J. E., Hrabak, E. M., Abe, M. \& Pankratz, S. H. (1984). Specific phases of root hair attachment in the Rhizobium trifoli-clover symbiosis. Appl Environ Microbiol 48, 1140-1150.

Finnie, C., Hartley, N. M., Findlay, K. C. \& Downie, J. A. (1997). The Rhizobium leguminosarum prsDE genes are required for secretion of several proteins, some of which influence nodulation, symbiotic nitrogen fixation and exopolysaccharide modification. Mol Microbiol 25, 135-146.

Finnie, C., Zorreguieta, A., Hartley, N. M. \& Downie, J. A. (1998). Characterization of Rhizobium leguminosarum exopolysaccharide glycanases that are secreted via a type I exporter and have a novel heptapeptide repeat motif. J Bacteriol 180, 1691-1699.

Glucksmann, M. A., Reuber, T. L. \& Walker, G. C. (1993). Genes needed for the modification, polymerization, export, and processing of succinoglycan by Rhizobium meliloti: a model for succinoglycan biosynthesis. J Bacteriol 175, 7045-7055.

Ho, S. C., Wang, J. L. \& Schindler, M. (1990). Carbohydrate binding activities of Bradyrhizobium japonicum. I. Saccharidespecific inhibition of homotypic and heterotypic adhesion. J Cell Biol 111, 1631-1638.

Ho, S. C., Wang, J. L., Schindler, M. \& Loh, J. T. (1994). Carbohydrate binding activities of Bradyrbizobium japonicum. III. Lectin expression, bacterial binding, and nodulation efficiency. Plant J 5, 873-884.

Hrabak, E. M., Urbano, M. R. \& Dazzo, F. B. (1981). Growthphase-dependent immunodeterminants of Rhizobium trifolii lipopolysaccharide which bind trifoliin A, a white clover lectin. $J$ Bacteriol 148, 697-711.

Jacobsson, K. \& Frykberg, L. (1995). Cloning of ligand-binding domains of bacterial receptors by phage display. Biotechniques $18,878-885$.

Jacobsson, K. \& Frykberg, L. (1996). Phage display shot-gun cloning of ligand-binding domains of prokaryotic receptors approaches $100 \%$ correct clones. Biotechniques 20, 1070-1076.

Jacobsson, K. \& Frykberg, L. (1999). Gene VIII-based, phage display vectors for selection against complex mixtures of ligands. In Expression Genetics: High-throughput Methods, pp. 225-238. Edited by M. McClelland \& A. B. Pardee. Natick, MA: Eaton Publishing.

Kijne, J. W., Smit, G., Diaz, C. L. \& Lugtenberg, B. J. (1988). Lectinenhanced accumulation of manganese-limited Rhizobium leguminosarum cells on pea root hair tips. J Bacteriol 170, 2994-3000.

Lilie, H., Haehnel, W., Rudolph, R. \& Baumann, U. (2000). Folding of a synthetic parallel beta-roll protein. FEBS Lett 470, 173-177.

Lodeiro, A. R., Lagares, A., Martinez, E. N. \& Favelukes, G. (1995). Early interactions of Rhizobium leguminosarum bv phaseoli and bean roots - specificity in the process of adsorption and its requirement of $\mathrm{Ca}^{2+}$ and $\mathrm{Mg}^{2+}$ ions. Appl Environ Microbiol 61, 1571-1579.

Loh, J. T., Ho, S. C., de Feijter, A. W., Wang, J. L. \& Schindler, M. (1993). Carbohydrate binding activities of Bradyrhizobium japonicum: unipolar localization of the lectin BJ38 on the bacterial cell surface. Proc Natl Acad Sci U S A 90, 3033-3037.

de Lorenzo, V., Herrero, M., Jakubzik, U. \& Timmis, K. N. (1990). Mini-Tn5 transposon derivatives for insertion mutagenesis, promoter probing, and chromosomal insertion of cloned DNA in gram-negative eubacteria. J Bacteriol 172, 6568-6572.

Maruyama, K., Mikawa, T. \& Ebashi, S. (1984). Detection of calcium binding proteins by ${ }^{45} \mathrm{Ca}$ autoradiography on nitrocellulose membrane after sodium dodecyl sulfate gel electrophoresis. J Biochem 95, 511-519.

Sambrook, J., Fritch, E. F. \& Maniatis, T. (1989). Molecular Cloning: a Laboratory Manual, 2nd edn. Cold Spring Harbor, NY : Cold Spring Harbor Laboratory.

Schäfer, A., Tauch, A., Jager, W., Kalinowski, J., Thierbach, G. \& Pühler, A. (1994). Small mobilizable multipurpose cloning vectors derived from the Escherichia coli plasmids pK18 and pK19 selection of defined deletions in the chromosome of Corynebacterium glutamicum. Gene 145, 69-73.

Sherwood, J. E., Vasse, J. M., Dazzo, F. B. \& Truchet, G. L. (1984). Development and trifoliin A-binding ability of the capsule of Rhizobium trifolii. J Bacteriol 159, 145-152.

Smit, G., Kijne, J. W. \& Lugtenberg, B. J. (1987). Involvement of both cellulose fibrils and a $\mathrm{Ca}^{2+}$-dependent adhesin in the 
attachment of Rhizobium leguminosarum to pea root hair tips. $J$ Bacteriol 169, 4294-4301.

Smit, G., Logman, T. J., Boerrigter, M. E., Kijne, J. W. \& Lugtenberg, B. J. (1989). Purification and partial characterization of the Rhizobium leguminosarum biovar viciae $\mathrm{Ca}^{2+}$-dependent adhesin, which mediates the first step in attachment of cells of the family Rhizobiaceae to plant root hair tips. J Bacteriol 171, 4054-4062.

Smit, G., Tubbing, D. M. J., Kijne, J. W. \& Lugtenberg, B. J. J. (1991). Role of $\mathrm{Ca}^{2+}$ in the activity of rhicadhesin from Rhizobium leguminosarum biovar viciae, which mediates the first step in attachment of Rhizobiaceae cells to plant-root hair tips. Arch Microbiol 155, 278-283.

Smit, G., Swart, S., Lugtenberg, B. J. \& Kijne, J. W. (1992). Molecular mechanisms of attachment of Rhizobium bacteria to plant roots. Mol Microbiol 6, 2897-2903.

Štyriak, I., Laukova, A., Fallgren, C. \& Wadström, T. (1999). Binding of selected extracellular matrix proteins to enterococci and Streptococcus bovis of animal origin. Curr Microbiol 39, 327-335.

Vasse, J. M., Dazzo, F. B. \& Truchet, G. L. (1984). Re-examination of capsule development and lectin-binding sites on Rhizobium japonicum 3I1B110 by the glutaraldehyde/ruthenium red/uranyl acetate staining method. J Gen Microbiol 130, 3037-3047.

Wisniewski, J.P. \& Delmotte, F. M. (1996). Modulation of carbohydrate-binding capacities and attachment ability of Bradyrhizobium sp. (lupinus) to white lupin roots. Can J Microbiol 42, 234-242.

Zorreguieta, A., Finnie, C. \& Downie, J. A. (2000). Extracellular glycanases of Rhizobium leguminosarum are activated on the cell surface by an exopolysaccharide-related component. J Bacteriol $182,1304-1312$.

Received 24 August 2000; revised 2 November; accepted 17 November 2000. 